
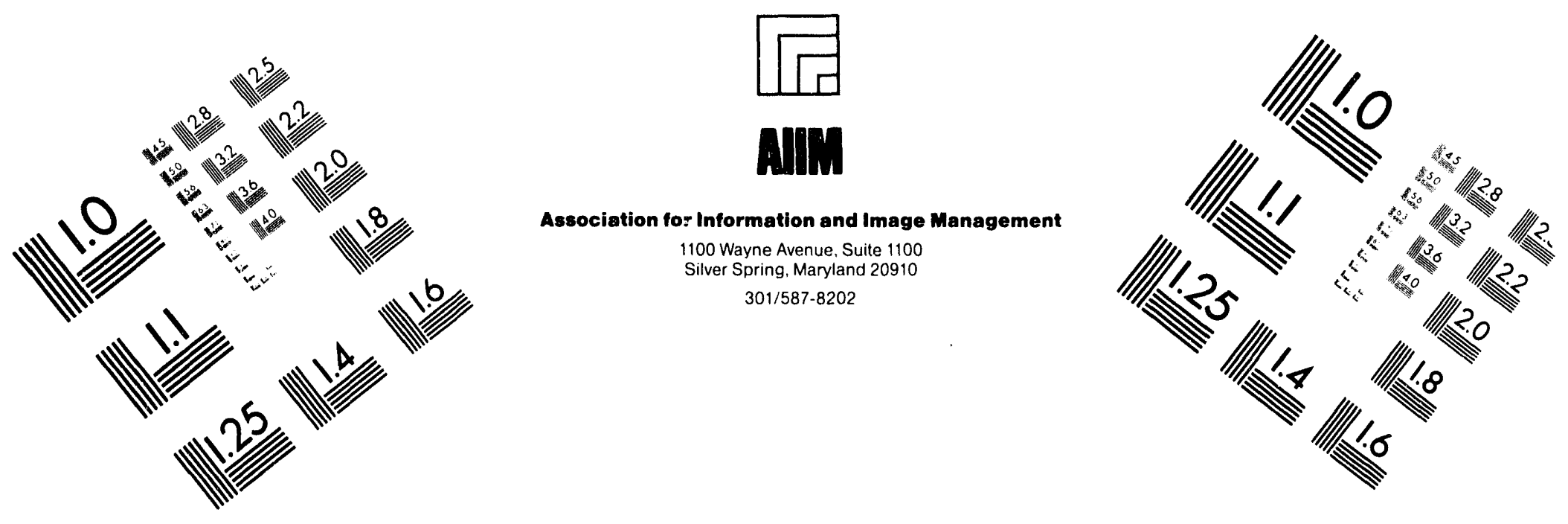

\title{
Centimeter
}

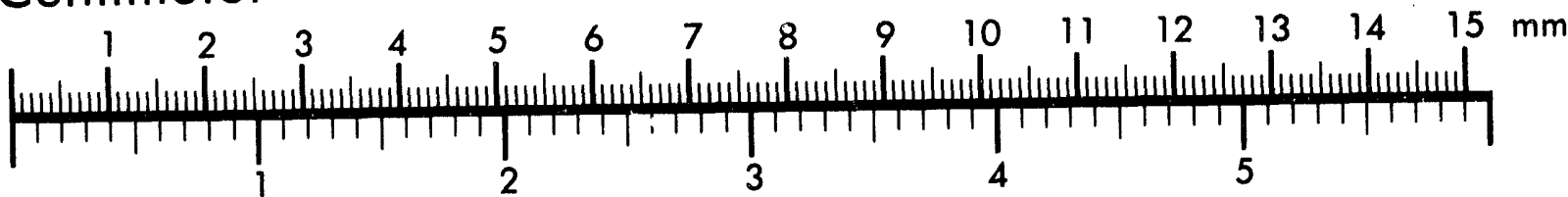
Inches
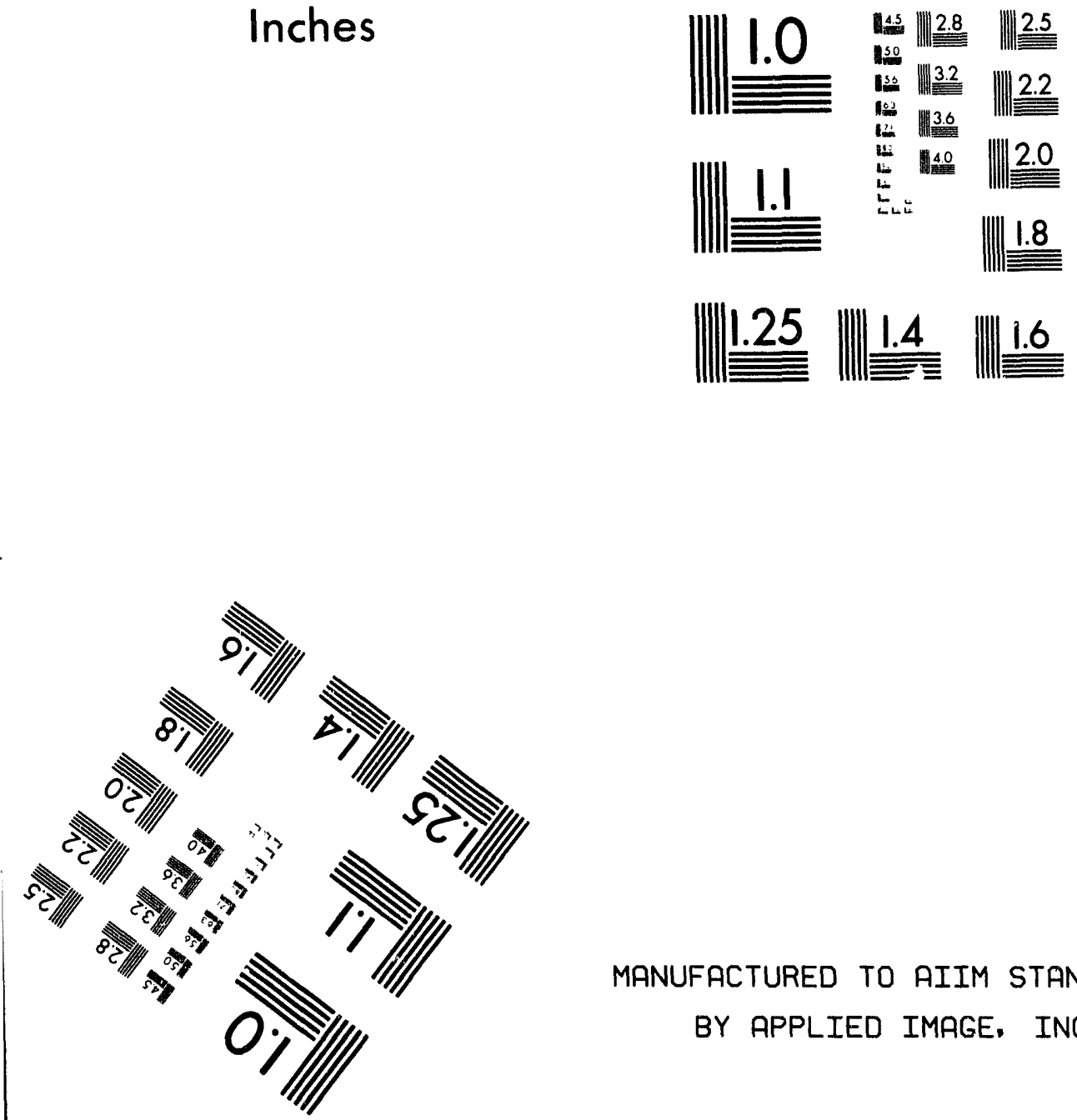

MANUFACTURED TO AIIM STANDARDS

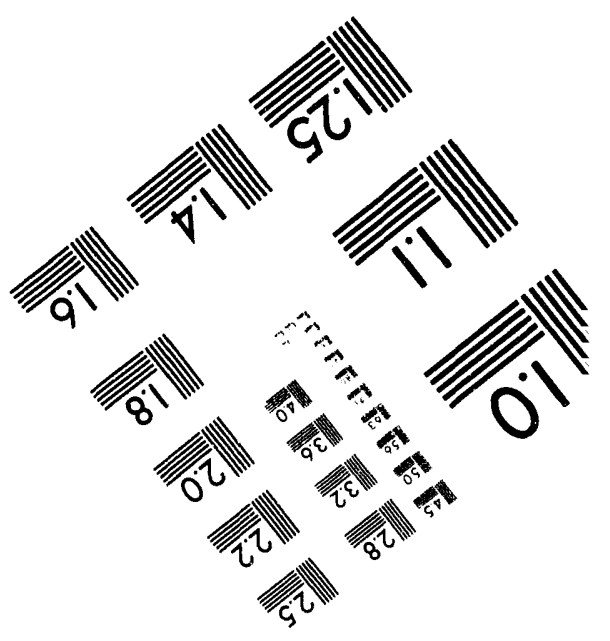



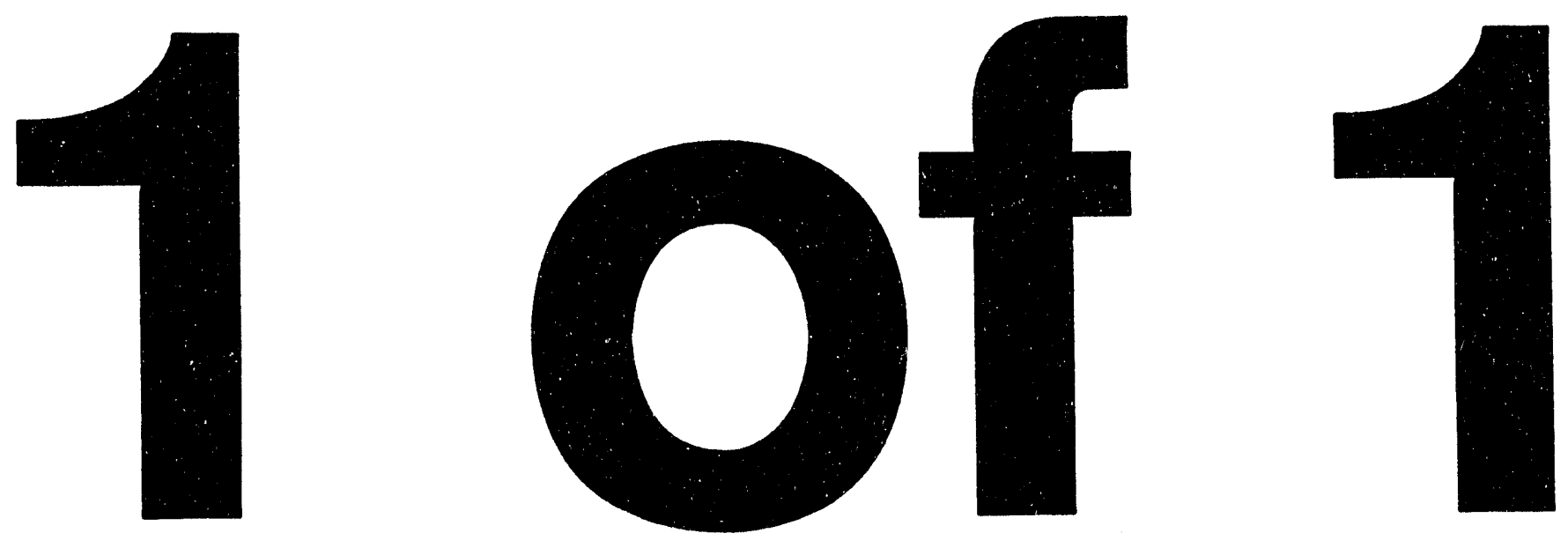
ARGONNE NATIONAL LABORATORY

ANL-94/11

9700 South Cass Avenue, Argonne, Illinois 60439

Distribution

Category:

Energy Research

(UC-

1400

\section{Solidification Modeling of a Spiral Casting to Determine Material Fluidity}

by

S. Ahuja, H. M. Domanus, and R. C. Schmitt

Energy Technology Division

and

L. Chuzhoy and J. V. Grabel

Caterpillar Inc.

Peoria, Illinois 61656

February 1994

Work sponsored by

U.S. DEPARTMENT OF ENERGY

Office of Industrial Technology, under Contract W-31-109-Eng-38

Caterpillar, Inc.

The State of Illinois 


\section{Solidification Modeling of a Spiral Casting to Determine Material Fluidity}

S. Ahuja, H. M. Domanus, R. C. Schmitt

Energy Technology Division

and

L. Chuzhoy and J. V. Grabel

Caterpillar, Inc., Peoria, IL

\section{Abstract}

In casting, fluidity is the measure of the distance a metal can flow in a channel before being stopped by solidification. During mold filling, the metal loses heat to the surrounding mold, thereby cooling and becoming more viscous until the leading portion solidifies and no further flow is possible. A coupled heat-transfer and fluid-flow modeling of a spiral, involving the use of thermophysical properties to determine material fluidity, has been conducted. Fluidity experiments were performed by Caterpillar; several spiral test castings were poured. Simulations of these experiments utilized the Casting Process Simulator (CaPS) software developed at Argonne National Laboratory. Two types of spiral geometries with different assumptions were considered: (1) a two-dimensional laterally stretched spiral and (2) a three-dimensional lateral spiral. The computed extent of mold filling is in good agreement with the experimental results. Time required by the metal/gas interface to attain specific positions in the spiral arm also compares favorably with the experimental results. 'The influence of process variables, especially pour time, is discussed. The CaPS software has been used as a computational tool to investigate the validity of the dimensionality assumptions and to evaluate the ability of CaPS to model fluidity adequately. 


\section{Contents}

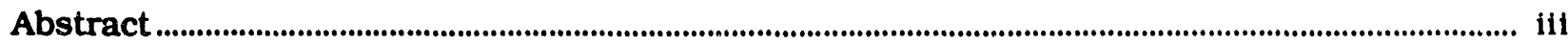

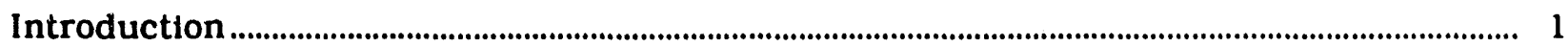

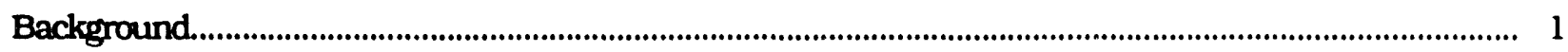

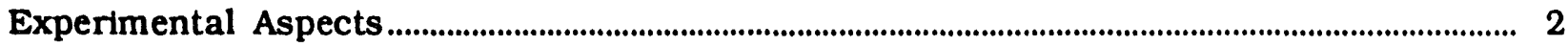

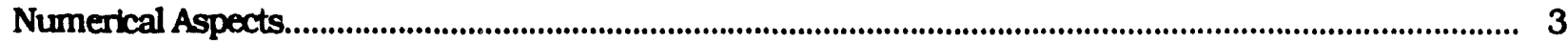

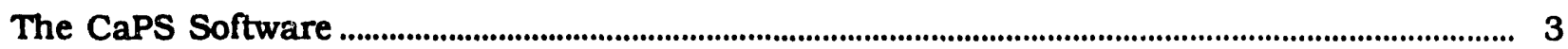

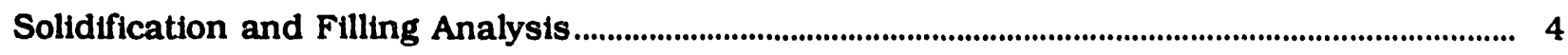

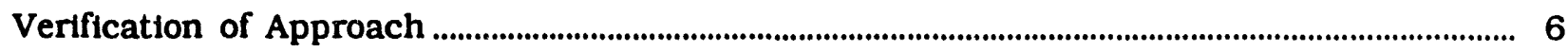

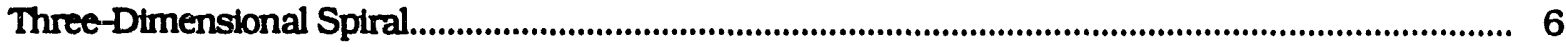

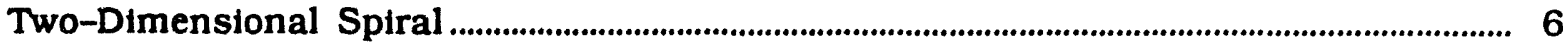

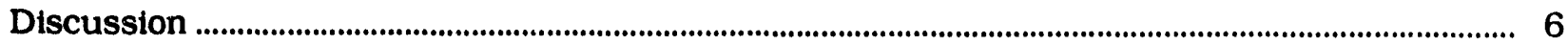

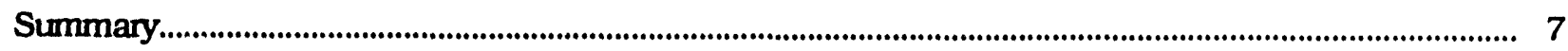

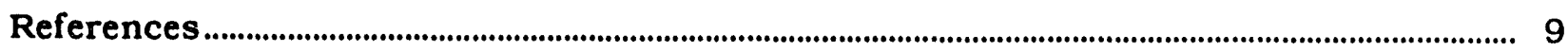

\section{Fugures}

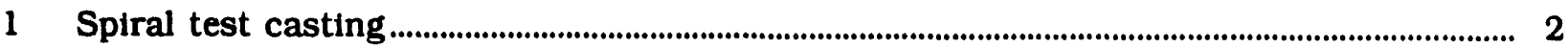

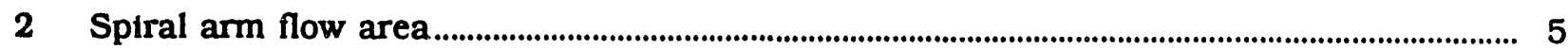

3 Spiral length versus pour time determined with CaPS ......................................................... 7

4 Experimental and simulated 2-D results of leading edge of metal/gas interface versus contact time.

5 Experimental and simulated 3-D results of leading edge of metal/gas interface versus contact time. 
Producing sound castings can be difficult. When liquid metal is poured into a mold. many processes begin: surface tension and microstructure change, metal penetrates sand and vice versa, turbulence and viscous effects ensue, etc. During solidification, the metal contracts, which in turn causes a pressure drop in the remnant liquid and regional buildup of excess solute, which produces segregation.

Most casting defects occur during initial pouring; therefore, the design of the running system, which guides the metal from the ladle into the mold, is crucial. The main aim of a mold designer is to produce sound, accurate, strong, and defect-free castings. The traditional design method for the running system and mold filling is a trial-and-error approach that is tedious, time consuming, and expensive. These difficulties can be overcome by a computer simulation that demonstrates the actual process of mold filling and subsequent solidification. Computer simulation of various processes has become increasingly common in recent years. The cost effectiveness of making flawless castings has made the foundry worker more aware of the process of mold filling, identification of hot spots, etc. There are several advantages of computer simulation over traditional casting methods: different geometries can be developed and tested in a short time, compared with the days of traditional trial-and-error methods to identify, for instance, position of risers and chill blocks, design of runners, etc.

In this paper, an effort is made to understand material nuidity via mold filling in a spiral test casting. The Casting Process Simulator (CaPS) software, ${ }^{1}$ developed at Argonne National Laboratory and based on transient heat transfer and fluid flow aspects of solidification, has been used to conduct computer simulations of the mold-filling process and the subsequent solidification of the metal in the mold. Experimental aspects have been demonstrated by Caterpillar, where several test castings were made to determine the repeatability and accuracy of results, primarlly with gray iron as the filling material in silica sand molds. Variations in experimental spiral lengths were modeled according to the duration of a liquid metal pour into the filling cavity.

\section{Background}

Several mold patterns have been suggested and used by researchers to identify the extent of mold filling by the molten metal, and consequently to determine fluidity. In most of the tests, the molten metal flows through a constricted channel that is long enough to extract sufficient heat to solidify the leading free surface of the metal and stop the flow of metal in the channel. Most of the tests have been conducted with a spiral channel ${ }^{2-4}$ that simplifies handling and leveling problems, although a laterally flowing channel has also been used. 5 The shape of the channel is either circular, semicircular, or trapezoidal to reduce surface contact of the metal with the mold.

The amount of metal that fills the mold depends on the test variables, e.g., mold pattern, pressure head, mold properties, superheat, pouring rate, etc.; and the properties of the metal, e.g., viscosity, oxide films, surface tension, etc. Because the viscosity of metals is a small multiple of that of water 6 and the kinematic viscosity (which is the viscosity divided by the mass density) is less than that of water, the existing effect of viscosity on metal fluidity is not very pronounced. The formation of oxide films reduces metal flow through the constricted channel and therefore may lead to decreased fluidity. Surface 
tension of metals increases with melting point and decreases with increase in temperature. 6 The effect of higher surface tension is to increase the pressure required to force the metal into the constricted channel and thus the spiral length is increased.

The design of the spiral test casting should be such that the system of gates in the mold pattern allows a constant pressure head and permits the metal to flow into the channel with negligtble inertia. The molding sand should be permeable so that gases emitted as a result of mold-to-metal contact can escape.

\section{Experimental Aspects}

Experimental investigations on the spiral test casting were conducted at Caterpillar. ${ }^{7}$ One purpose of the tests was to confirm the reliability and repeatability aspects of the spiral test castings. The basic experimental casting design is shown in Fig. 1; it is similar to that used in earlier research. 4 The experimental setup consisted of a pour basin to create a constant pressure head for the liquid metal flowing into the channel, a downgate with a constricted opening at the base to limit the amount of the metal flowing intc the channel, a pool to dissipate the kinetic energy of the falling metal, and the spiral arm. A reservoir in the pour basin stabilized the flow of metal into the downgate. Every effort was made to ensure constant conditions during the experiments.

The pour basin and the downgate patterns in the cope were connected via an orificed core around the base of the downgate to the drag of the mold, which consisted of the pool and the spiral patterns. The cope was $3.0 \mathrm{ln}$. high and the drag was $4.0 \mathrm{in}$. deep. The spiral arm was curved so that the adjacent arms were spaced $0.55 \mathrm{in}$. apart. The thickness and depth of the arm were 0.36 and 0.3 in., respectively, and the shape was a half-circle at the base so that surface contact between the metal and the mold was limited. The pool was $2.5 \mathrm{in}$. deep and $1.25 \mathrm{in}$. in diameter, the orifice of the core was $0.375 \mathrm{in}$., and the downgate was 1.75 in. tall.

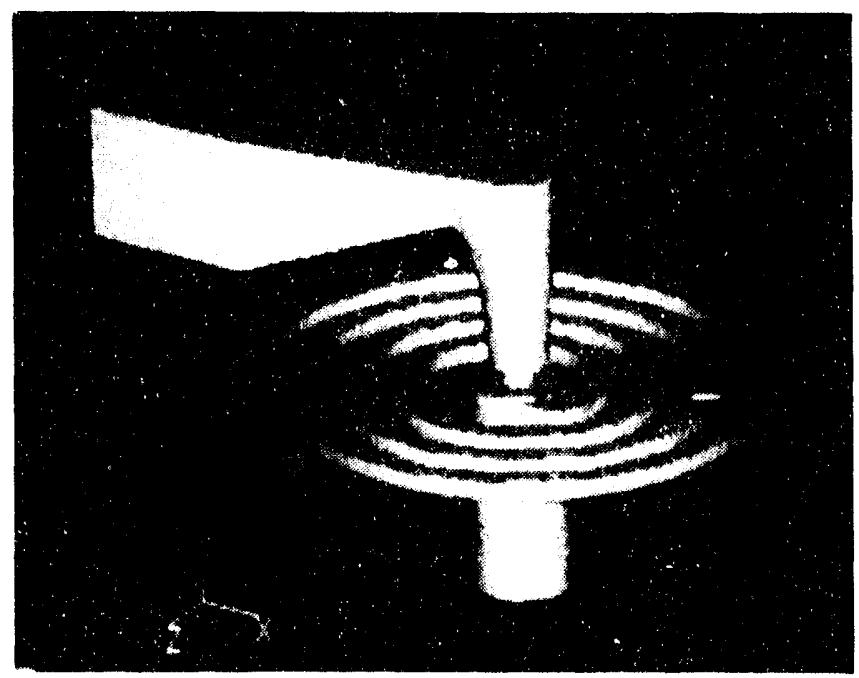

Fig. 1. Spiral test casting 
Twenty-six spiral test castings were poured: 12 for Group 1 and 14 for Group II. The tests consisted of spiral test castings poured under identical conditions and constant processing variables. Group I castings were poured from four ladles with three heats poured per ladle. Pouring temperature was accurately determined at the beginning and end of each pour, and the superheat was maintained as constant as possible. Metal properties were monitored by pouring the liquid metal into a circular, button-shaped mold during each tap to obtain the chemical composition of the melt. The time from start of melt to pour was recorded for each ladle. Further, because the spiral length depends upon the pressure of the liquid metal flowing through the channel, the distance between the ladle lip and the pour basin was kept constant.

Five of the 14 Group II castings were equipped with unconnected K-type thermocouple wire pairs. The pairs were placed $0.25,9.0,14.0,19.0,24.0$, and $29.0 \mathrm{in}$. from the beginning of the spiral arm. The thermocouple wires were inserted in the mold through a diametrically consistent opening. Only the uninsulated bare wire protruded into the metalfilling cavity, and the opening was sealed with silicon paste. When liquid metal reached the thermocouple wires, a circuit was completed and a signal sent to a computer to record the time (in tenths of seconds) of metal contact with the sensors. Five ladles were used to make three castings each.

Experimental spiral length ranged from 20 to $26.25 \mathrm{in.}$, with an average of 23.27 in. The repeatability of time of metal contact with the sensors was good, with a deviation of $<10 \%$ of the mean. Each of the three molds from individual ladles evolved similar spiral lengths. The variation of the spiral length was more pronounced when different ladles were used.

\section{Numerical Aspects}

With the advent of powerful workstations, modeling of heat transfer and fluid flow aspects of solidification has become increasingly efficient. Vartous models have been proposed for either heat transfer, fluid flow, or combined aspects during mold filling. microstructural evolution, etc. ${ }^{8}$. Other researchers have worked on treating surface boundary conditions with inherent mold-to-metal heat transfer properties. 9 This avoids including the mold in the geometry, leading to fewer cells in the geometry and thus time savings in computation time; however, it deters the user from making quick simulation runs because the setup time of a simulation run for a complex casting is large.

The objectives of numerical modeling of any solidification aspect are to obtain good agreement between experiments and theory, minimize computation time, and allow the foundry worker to use the model with minimum input parameters and help in simulating reality. Again, as the geometries become more complex, the number of cells needed to mesh the geometry becomes large, thereby increasing computational time.

\section{The CaPS Software}

The macroscopic Casting Process Simulator (CaPS) software combines heat transfer and fluid flow aspects and can describe various solidification aspects, including mold filling. CaPS is a multidimensional time-dependent computer code that uses a finite-volume formulation for mass, momentum, and energy equations. In CaPS, the geometry is created independently of the mesh, using PATRAN, which is based on grids and line 
representation, and utilizing patches to realize surfaces and hyperpatches to create geometric volumes. CaPS reads the neutral file, which consists of a list of named components. The geometry is meshed with a structured mesh generator of regular cells interfaced with the neutral file output of the solid geometric package. Visual user interfaces have been developed on the basis of the HOOPS hierarchical libraries, which contain a database of geometric information. These visual interfaces allow the user to create, observe, verify, and view the meshed geometry, set up the boundary and initial conditions, view the different named components, and initialize the simulation parameters.

The CaPS shell scripts interactively provide a step-by-step procedure to simulate a solidification aspect, thus making the software user-friendly. With CaPS, an efficient solution of transient fluid flow and heat conduction within the metal and between the metal and the mold can be realized.

\section{Solidification and Filling Analysis}

Basically, two underlying concepts are used by researchers to describe a free surface. In the Lagrangian approach, although the boundary conditions are easily applied, the analysis breaks down when large surface distortions are present and when two or more surfaces intersect. CaPS employs the Eulerian approach, which makes use of the volumeof-fluid concept 10 to analyze free surfaces efficiently and generate approximations for convective fluxes.

The rapidly transient fluid flow consists of the liquid metal flowing through runners and gates into the cavity in a complex manner involving intersecting free surfaces. In CaPS, the volume-of-fluid concept is realized by classifying each cell as either empty or partially filled. The new velocity field is calculated on the free-surface boundary, where pressure is specified. The amount of fluid is computed when the cell empties or fills. Also, if the cell cannot be resolved by free-surface boundary conditions on velocity, the process is iterated. The governing equations are the basic equations of conservation of mass, momentum, and energy. The calculation of mass fraction is based on the enthalpy method. The solid mass fraction, $x_{\mathbf{s}}$, is given by

$$
\mathbf{x}_{\mathrm{s}}=\left(\mathrm{h}_{\mathrm{l}}-\mathrm{h}\right) /\left(\mathrm{h}_{\mathrm{l}}-\mathrm{h}_{\mathrm{s}}\right) \text {. }
$$

where $h$ is the enthalpy and the subscripts 1 and $s$ denote liquidus and solidus, respectively. The latent heat released during solidification is inherently involved in the temperaturedependent properties. In CaPS, thermal conductivity, density, viscosity, and specific heat are to be specified as a function of temperature for each material used in the experiments.

The geometry is meshed separately and is not inherently related to boundary conditions, as prescribed by some researchers. ${ }^{9}$ Thus, the user is not required to specify each section of the casting boundary. Computation time can be reduced by reducing the width of the mold in the peri- hery of the casting. thus reducing the number of cells in the mold material. In the CaPS code, the pressure solver relates only to the cavity of the geometry. Thus, computational time for the entire mold-cavity assembly is used efficiently in CaPS.

A force structure was used to model the momentum interaction between the liquid and the mold in the spiral arm. A force structure is a mechanism whereby a drag or resistance 
force (in $\mathrm{Pa} / \mathrm{m}$ ) can be applied to a fluid flow across a cell face between two computational cells.

A thermal structure model has been used to model the heat transfer interaction between the molten metal and the mold in the spiral arm. Thus, while creating the geometry of the spiral arm, the mold was neglected. This drastically reduced the number of computation cells and thereby computation time. The thermal-structure model in CaPS solves one-dimensional heat conduction equations for all solid thermal structures. It calculates temperature distribution in solids and heat transfer from solids to surrounding fluids. In summary, the output from the thermal-structure model is a heat source/sink term for the fluid energy equation.

To calculate heat transfer between the fluid and solid surfaces (the mold, in particular), a heat-transfer-coefficient model is required. A thermal structure is a solid structure in a fluid-flow domain undergoing heat transfer interaction with the surrounding fluid. Thus, to model the heat transfer between the metal and the mold, plane thermal structures were included as auxiliary inputs. The heat transfer coefficient, $h$, is given by

$$
\mathbf{h}=\mathbf{k ~ N u} / \mathrm{D}_{\mathrm{E}}
$$

where $D_{E}$ is the characteristic length. $\mathrm{Nu}$ is the Nusselt number, and $\mathrm{k}$ is the thermal conductivity of the liquid metal. The Nu correlation used was

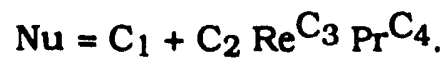

Here, Re is the Reynolds number; $P r$ is the Prandtl number; $C_{1}, C_{2}, C_{3}$, and $C_{4}$ are the constant coefficients for a given correlation; and $\mathrm{k}$ is the thermal conductivity of the liquid. The values used are $C_{1}=0.4, C_{2}=0.625, C_{3}=0.4$, and $C_{4}=0.4$. Also,

$$
\operatorname{Re}=\rho * U * D_{E} / \mu, \quad \text { and } \quad \operatorname{Pr}=\mu * C_{p} / k \text {. }
$$

where $\rho$ is the density, $U$ is the velocity, $\mu$ is the viscosity of the liquid metal, and $C_{p}$ is the specific heat of the liquid. The actuai cross-sectional area for flow, according to Fig. 2 , is $\mathrm{A}_{c}$ $=0.0940938 \mathrm{in} .^{2}$. The wetted perimeter is $w_{p}=1.16549 \mathrm{in}$. The equivalent hydraulic diameter, $D_{E}=4 A_{c} / w_{p}=0.3229$ in. Further, the equivalent depth is $A_{c} / 0.36=0.26137$ in. Thus, to approximate the semicircular spiral arm to a rectangular spiral arm, so that the flow area is consistent, a width of $0.36 \mathrm{in}$. and a depth of $0.26137 \mathrm{in}$. were used.
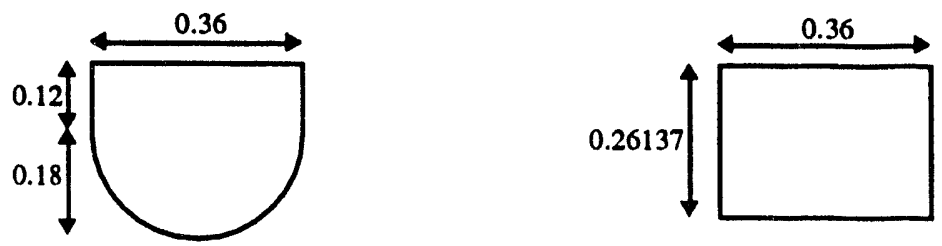

Fig. 2. Spiral arm flow area 
The numerical CaPS software was tested by varying the pour time of the liquid metal into the filling cavity via the spiral test casting. The simulations were conducted in two and three dimensions. A symmetric plane was created in the three-dimensional (3-D) lateral spiral to decrease the number of cells in meshing the geometry, thereby decreasing computation time and easing the geometric view. In both the two-dimensional (2-D) and 3-D spiral-arm geometries, a drag force in the $x$-direction was considered. The simulations are described in detail below.

\section{Three-Dimensional Spiral}

As indicated by earlier researchers, 5 a lateral spiral arm may be created if leveling is not a problem. Thus, to create a symmetric plane, the geometry of the spiral arm was made lateral because leveling is not a problem in computer simulations. The geometry was created according to the experimental spiral-cast dimensions furnished by Caterpillar. The elevated pour basin allowed simulation of the constant distance between the ladle lip and the pour basin.

In the symmetric 3-D model, the length of the spiral arm was 32 in., the width was $0.18 \mathrm{in}$. due to symmetry, and the depth was $0.26137 \mathrm{in}$. These dimensions created the same flow area as in the original pattern, which included a semicircular spiral arm. The entire geometry that is simulated and meshed involves the mold as an overlapping entity to the pour basin, downgate, and pool. Automatic timestepping was selected for the runs. The liquid metal enters from the inlet at a specified rate over a time interval. The temperature of the liquid metal was $1395^{\circ} \mathrm{C}$ and the initial mold temperature was $20^{\circ} \mathrm{C}$. The liquid metal begins to flow into the spiral arm after filling the pool. A cold-shut is caused by cooling of the leading edge of the flowing metal.

\section{Two-Dimensional Spiral}

The geometry of the laterally stretched 32-in. spiral consisted of an inlet, a pour basin, a downgate, a pool, and the spiral arm. This setup enabled viewing of the liquid metal as it flowed through the downgate into the pool, filled the pool, and entered the spiral arm. The meshed geometry of the 2-D lateral spiral again consisted of a peripheral mold along the pour basin, the downgate and the pool. As in the 3-D model, the elevated pour basin allows simulation of the actual experimental distance between the ladle lip (here, the inlet) and the pour basin. The metal stops flowing into the cavity when the temperature of the leacing edge falls below the solidus temperature and a cold-shut is created.

\section{Discussion}

The chemical composition, determined via a chemical button after each run, indicated very slight variation. The recorded time from start of melt to pour was constant, as was the distance between the ladle lip and the pour basin. The pouring temperature and the superheat were maintained as constant as possible. Thus, all experimental variables were kept as constant as possible. 


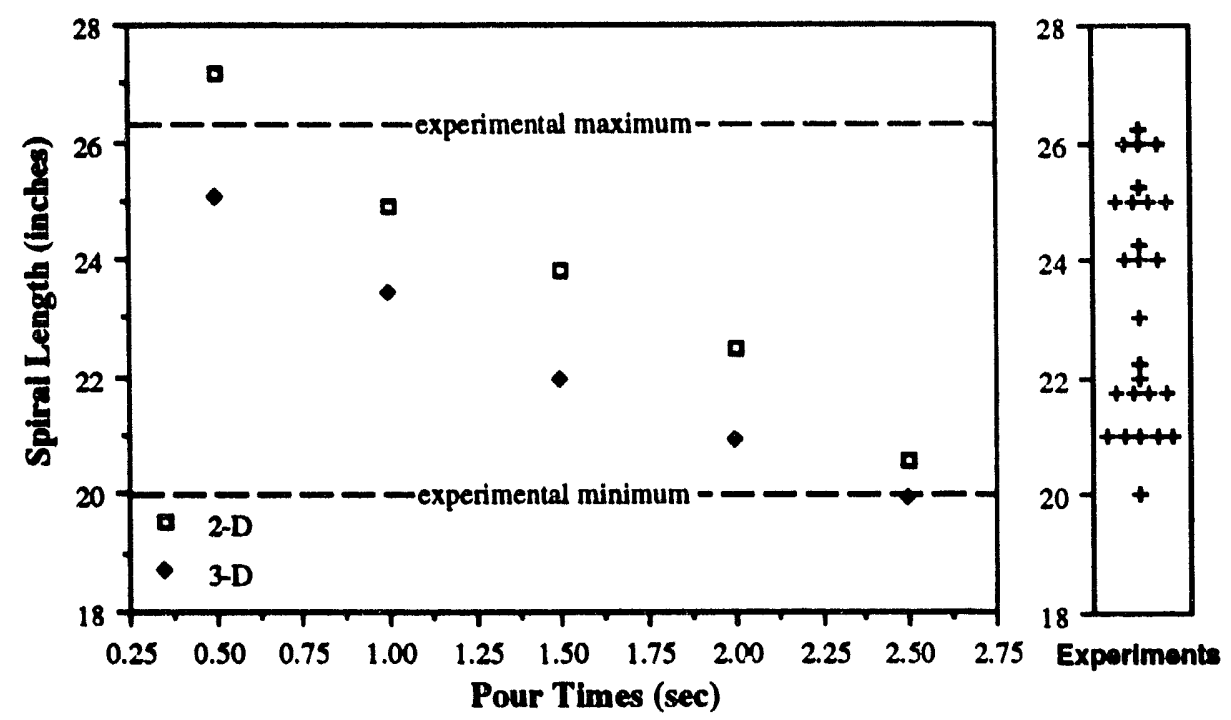

Fig. 3. Spiral length versus pour time determined with CaPS; experimental results are included at right

One variable subject to human variation was pour time. This variable could potentially indicate a variation of $6.25 \mathrm{in}$. (minimum of $20 \mathrm{in}$. and maximum of $26.25 \mathrm{in}$.) in the length of the spiral arm. To vary the pour rate, a transient-velocity boundary condition was used at the inlet. The velocity was assumed constant during the pour time interval and then set to zero. In all cases, the same amount of metal was poured. Figure 3 is a graph of various pour times versus spiral length determined with CaPS. The experimental portion is also appended on the right-hand side. Results of both the 2- and 3-D versions of the spiral geometry are displayed.

The 3-D simulation appears to be more sensitive to pour time than is the 2-D simulation. This is due to more accurate modeling of the pour basin, downgate, and pool in three dimensions. The variation of pour times appears to span the spiral lengths, as observed in the experiments. Thus, pour time may be an important criterion to be controlled during experiments.

The times required for the metal to reach certain points in the spiral arm were calculated relative to the time at which the liquid metal reached the pool bottom. Figure 4 shows the 2-D experimental results at the metal/gas interface versus contact times. Figure 5 is the corresponding 3-D plot. The simulation plots at a pour time of $2.5 \mathrm{sec}$ are included in both figures. The experiments were conducted with the minimal pour time, and it appears that the pour time of $2.5 \mathrm{~s}$ is in agreement with experimental results.

\section{Summary}

An increased understanding of fluidity has been achieved with the CaPS code and computer simulations of the mold-filling and subsequent solidification process. CaPS is a multidimensional time-dependent computer code that can predict how a liquid metal is gravity-fed into a sand mold through gates and runners, how the temperature varies in and around the casting, and how the metal fills and then solidifies in the cavity. Mold-heating effects can also be visualized. Simulations were conducted on two types of geometries: (1) a 


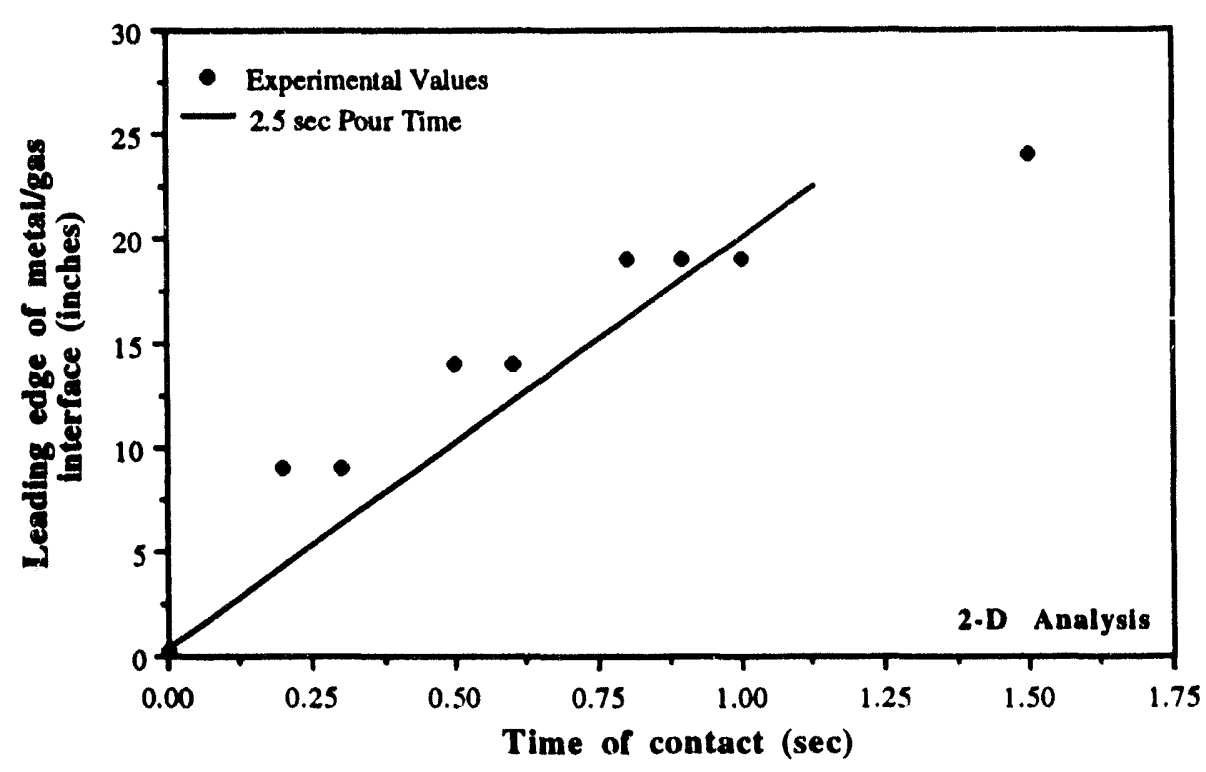

Fig. 4. Experimental and simulated 2-D results of leading edge of metal/gas interface versus contact time

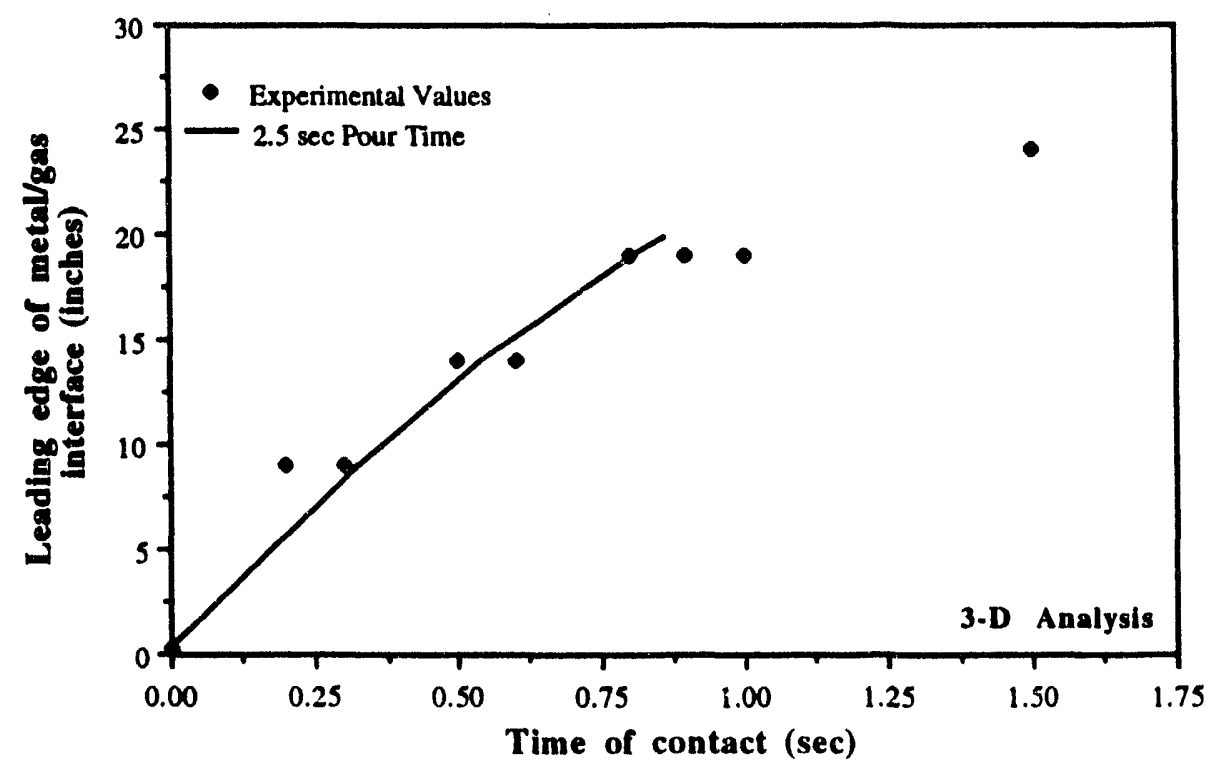

Fig. 5. Experimental and simulated 3-D results of leading edge of metal/gas interface versus contact time 
two-dimensional lateral spiral and (2) a three-dimensional lateral spiral. Molds with and without sensors were used in the experiments.

The simulation time of sensor contact with the flowing metal agreed with the experimental values. While great care was taken to maintain the process variables as constant as possible, a 6.25-in. variation in spiral length was observed experimentally. These results compare favorably with the simulation results, demonstrating that if leveling and handling are not significant factors, the lateral spiral test casting can also be used to conduct fluidity tests. While performing the simulations, we realized that variation in pour time may explain the observed 6.25-in. variation in experimental spiral length. Simulations with CaPS indicate that pour times of 0.75-2.5 $\mathrm{s}$ may be responsible for the varying spiral lengths. Indeed. It appears reasonable that manually, pour time is difficult to control accurately to within fractions of a second.

In the development of castings, mold filling is important. Free-surface modeling gives better insight into the casting process and can improve casting quality while reducing costs by minimizing scrap. With CaPS, mold-filling techniques can be varied by changing the pcsition of asers or gates to reduce the formation of shrinkage cavities. Also, rapid msld filling. which is important for thin-sectioned castings where increased heat loss results in premature freezing. can be performed by optimizing the gating design. CaPS is a software tool that permits foundries to save time and energy while reducing casting scrap.

Other aspects of solidification can also be readily simulated with the CaPS software. The effect of surface tension on the flow of liquid metal through the cavity, microstructural features of the final cast part, etc., will be incorporated to make the software even more useful to the casting industry.

\section{References}

1. H. M. Domanus, R. C. Schmitt and S. Ahuja, User's Guide for the Casting Process Simulator Software CaPS-2D, Version 1.0. Argonne National Laboratory Report ANL93/14 (July 1993).

2. C. M. Saeger and A. Krynitsky, A Practical Method for Studying the Running Quality of a Metal Cast in Foundry Molds, Trans. Am. Foundrymen's Soc., 39 513-540 (1931).

3. H. F. Taylor, E. A. Rominski, and C. W. Briggs, Fluidity of Ingot Iron and Carbon and Alloy Cast Steels, Trans. Am. Foundrymen's Soc., 29 1-93 (1941).

4. L. P. Porter and P. C. Rosenthal, Factors Affecting Fluidity in Cast Iron. Trans. Am. Foundrymen's Soc., 60 725-735 (1952).

5. W. Ruff. The Running Quality of Liquid Malleable Iron and Steel, Iron Steel Inst., 25 139 (1936).

6. CRC Handbook of Chemistry and Physics, 72nd Ed., CRC Press, Inc., (1991-92).

7. M. C, McNett, Caterpillar, Inc., Inter-Report, private communication (1991).

8. Metals Handbook, 9th Ed., ASM International, 15 (Casting) 855-891 (1988). 
9. J. A. Dantzig and S. C. Lu, Modeling of Heat Flow in Sand Castings: Part I. The Boundary Curvarure Method. Met. Trans., 16B 195-202 (1985).

10. C. W. Hirt and B. D. Nichols, Volume of Fluid (VOF) Method for the Dynamics of Free Boundaries, J. Computational Phys., 39 201-225 (1981) 
Distribution for ANL-94/11

Internal:
S. Ahuja (25)
C. A. Malefyt
R. C. Schmitt (5)
H. M. Domanus (5)
R. B. Poeppel
R. A. Valentin
H. Drucker
W. W. Schertz
R. W. Weeks
TIS Files

\section{External:}

DOE/OSTI (2)

ANL Librartes

ANL-E (2)

ANL-W

Manager, Chicago Field Office, DOE

Energy Technology Division Review Committee:

H. K. Birnbaum, Untversity of Illinois, Urbana

R. C. Buchanan, Untversity of Cincinnati, Cincinnati, $\mathrm{OH}$

M. S. Dresselhaus, Massachusetts Institute of Technology. Cambridge, MA

B. G. Jones, University of Illinois, Urbana

C.-Y. Li, Cornell University, Ithaca. NY

S.-N. Liu, Fremont, CA

R. E. Smith, SciTech, Inc., Morrisville, NC

Caterpillar, Inc., Peorla, IL

L. Chuzhoy

J. V. Grabel

M. Pershing 

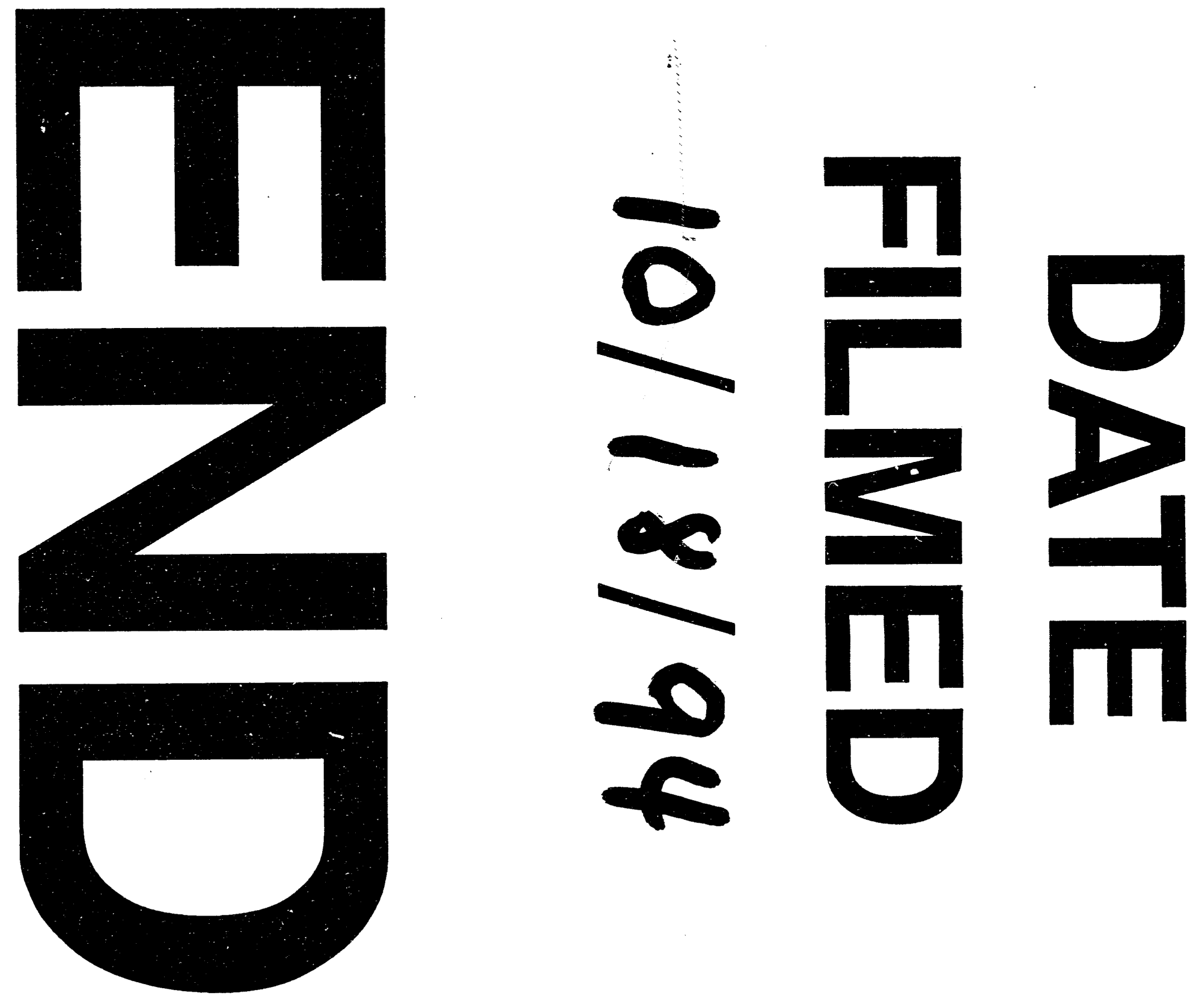
